\title{
Übersetzung pejorativer Personenbezeichnungen
}

\author{
Anita Pavić Pintarić (Zadar)
}

\begin{abstract}
This paper investigates the translation of pejoratives referring to persons. The corpus is comprised of literary dialogues in the collection of short stories about the First World War by Miroslav Krleža. The dialogues describe the relationship between officers and soldiers. Soldiers are not well prepared for the war and are the trigger of officers' anger. Therefore, the dialogues are rich with emotionally loaded outbursts resulting in swearwords.

Swearwords relate to the intellect and skills of soldiers, and can be divided into absolute and relative pejoratives. Absolute pejoratives refer to the words that carry the negative meaning as the basis, whereas relative pejoratives are those that gain the negative meaning in a certain context. They derive from names of occupations and zoonyms. The analysis comprises the emotional embedment of swearwords, their metaphoric character and the strategies of translation from the Croatian into the German language.
\end{abstract}

\section{$1 \quad$ Einleitung}

In diesem Beitrag wird die Übersetzung ${ }^{1}$ der pejorativen Personenbezeichnungen in der Novellensammlung Hrvatski bog Mars ,Der kroatische Gott Mars' von Miroslav Krleža untersucht. ${ }^{2}$ In literarischen Texten werden mit Pejorativa häufig das Verhalten oder Intellekt der Figuren bewertet (vgl. Pavić Pintarić, in Druck). Pejorativa werden in den Dialogen verwendet, die als Nachahmung der gesprochenen Kommunikation dienen. ${ }^{3}$

\footnotetext{
${ }^{1}$ Die Übersetzung Der kroatische Gott Mars. Erzählungen. (Königstein: Athenäum) aus dem Jahre 1984 stammt von Milica Sacher-Masoch, Reinhard Federmann und Milo Dor.

${ }^{2}$ Dieser Aufsatz kann als Folge des Aufsatzes zur Emotionalität in Krležas Novellen (Pavić Pintarić, in Druck) betrachtet werden. Die vier Novellen in dieser Sammlung beziehen sich auf den Ersten Weltkrieg. „Die Schrecken des Krieges, die blinde Dummheit der Militärs werden quer durch das Leiden und den Verfall der Menschen aufgezeigt. Blut, Eiter, Schleim, stechender Schmerz - und als Gegengewicht ein Aufbäumen der Instinkte. - In den Sanitätsstationen an der Front stürzen sich Syphilitiker auf Obszönitaten, Fotos von Verwundeten, Alkohol und die Krankenschwestern, während der Direktor - Herr Graf - und die Herren Offiziere und Ärzte sich bei feindlichen Offensiven zurückziehen und die Leute sich selbst überlassen." (Claude Bonnefoy, http://www.kriticnamasa.com/item_de.php?id=840 [25.10.2016]).

${ }^{3}$ Dafür wird in der Forschung der Begriff ,fingierte Mündlichkeit‘ (Goetsch 1985) oder ,fiktive Mündlichkeit ${ }^{\star}$ (vgl. z. B. Rocco/Pavić Pintarić 2016) verwendet.
}

Linguistik online 82, 3/17 - http://dx.doi.org/10.13092/lo.82.3718

CC by 3.0 
Pejorative Personenbezeichnungen werden im vorliegenden Beitrag als Ausdrücke der emotiven Funktion der Sprache in einem literarischen Werk betrachtet. ${ }^{4}$ Laut Pavić Pintarić (in Druck) zeigt sich in den Novellen von Krleža, die den ersten Weltkrieg thematisieren, dass Soldaten durch Pejorativa als dumme und schmutzige Menschen sowie Nichtsnutze bewertet werden. Figuren von Vorgesetzten, Offiziere, verwenden Pejorativa, um auf diese Weise ihren Ärger abzureagieren. Bergerová (2015: 136-137) sieht Schimpfen als die vierte Phase unter den fünf Phasen des prototypischen Ärger-Szenarios. In der vierten Phase stellt sie folgende Verhaltensmuster dar: Verlust der Kontrolle, Herauslassen des Ärgers (Drohen, Schimpfen, Fluchen, Sich-Beschweren oder gewalttätiges Verhalten), die Mimik, Gestik und Motorik begleiten. Die emotive Funktion sieht Liimatainen (2011: 122-123) in sog. ,Fluchund Invektivformeln' mit absichtlich beleidigendem Charakter (Verfluchter Mist!, Hol's der Henker! Gottverdammter Idiot!).

Herauslassen des Ärgers wird in den Dialogen durch Ausrufesätze mit expressiver syntaktischen Struktur ausgedrückt, d. h. den pejorativen Personenbezeichnungen werden Personalund Demonstrativpronomen sowie negativ konnotierte attributive Adjektive (v. a. faul, lausig, verdammt) nachgestellt. Pejorativ bezeichnete Personenbezeichnungen stammen vor allem aus den Bereichen der Berufe und Zoonyme.

Es wird in diesem Beitrag davon ausgegangen, dass Pejorativa zugleich Metaphern und Werturteile sind, d. h. dass sie eine emotionalisierende Funktion haben, indem sie zur Beleidigung verwendet werden. In der Analyse werden Personenbezeichnungen aus dem Ausgangstext (AT) als emotionale Ausdrücke betrachtet. Des Weiteren werden ihre Bedeutung und der Kontext, in dem sie benutzt werden, untersucht. Nach der Analyse der Pejorativa im AT werden Pejorativa im Zieltext (ZT) untersucht und Übersetzungstechniken analysiert. Die Übersetzungsanalyse umfasst folgende Punkte: a) Werden im Zusammenhang mit den Pejorativa im Text Emotionen ausgedrückt? b) Werden Pejorativa aus dem AT mit entsprechenden Wörtern im ZT übersetzt? c) Ist das tertium comparationis im AT und ZT gleich? d) Wird die Intensität der Beleidigung aus dem AT im ZT beibehalten?

\section{Pejoration im Deutschen und Kroatischen}

Pejoration bezieht sich auf „das Abgleiten in eine abwertende, negative Bedeutung“ (DUW5 2003: 1192), die zugleich mit allen Ebenen der Sprachbeschreibung verbunden ist (Stojić/Pavić Pintarić 2014: 345). Pejorativa werden als Ausdruck von Verachtung, Minderwertigkeit oder Hass verwendet, um bestimmten Gruppenmitgliedern zu schaden (vgl. Croom 2013). Pejorativa können absolut und relativ sein (vgl. Stojić/Pavić Pintarić 2014: 347): Absolut sind diejenigen, die die negative Konnotation in ihrer Grundbedeutung behalten (z. B. Dummkopf/glupan, Narr/budala), während relative Pejorativa ihre negativen Konnotationen erst in einem bestimmten Kontext realisieren, z. B. Ochse/vol, Schwein/svinja. So

\footnotetext{
${ }^{4}$ Der abwertende Gebrauch bestimmter Lexeme impliziert die emotionale Aufladung und damit eine Einstellung zum Genannten. Diese Einstellung wird in der Bewertungstheorie nicht explizit als eine pejorative Kategorie bezeichnet. Martin und White (2005: 73-74) nennen einige Beispiele des negativen Urteils, die Beschimpfungen umfassen, z. B. you lobotomised moron, wo das Adjektiv das pejorative Substantiv noch verstärkt, wie auch in (you complete moron, you total idiot, you absolute imbecile, etc.). Espunya und Pavić Pintarić (2016: 136) sind der Meinung, dass Pejorativa der Kategorie ,Urteil', insbesondere der ,Normalität‘ zuzuordnen sind, da „Stereotype Kategorisierungen aufgrund erkannter Unterschiede umfassen“.

5 Duden Deutsches Universalwörterbuch.
} 
nennt O'Brien (2009: 35) Metaphern der Organismen, der Tiere, des Krieges, der Religion oder der Gegenstände, die negativ konnotiert werden können und die eine Gruppe von Menschen dehumanisieren oder sie als eine Gefahr für die Gesellschaft beschreiben können. Das negativ Konnotierte in der Kommunikation führt häufig zur Aufregung der Kommunikationspartner und spielt deswegen in der Kommunikation eine wesentliche Rolle; dazu sind negativ konnotierte Ausdrücke bildhaft und kreativ (Hayryliv 2009: 33).

Havryliv (2009: 49) teilt Pejorativa in ihrer Untersuchung des Wienerischen in metaphorische und metonymische Pejorativa ein. Bei metaphorischen Pejorativa werden zwei Größen miteinander nach einer gemeinsamen Eigenschaft verglichen, d. h. nach dem tertium comparationis oder dem Dritten des Vergleichs. Havryliv (ebd.: 37) spricht auch von universalen Pejorativa, die „eine allgemeine negative Charakteristik des Adressaten“ und die negative Stellungnahme zum Adressaten liefern und sich gesondert auf Frauen und Männer beziehen (z. B. Miststück, Sauweib, blöde Kuh oder Scheißkerl, Arschloch, Dreckskerl, Mistkerl). Beller (2013: 136) unterscheidet folgende Faktoren, die Pejorativa von anderen Substantiven unterscheiden: Sie basieren auf Generalisierung der bemerkbaren, typischen Verhaltensmerkmale; sie drücken subjektive Bewertungen aus; die Bewertungen können graduiert werden. Scheffler (2000: 105) hebt die Struktur der Beschimpfungen hervor: Personalpronomen + Adjektiv + Schimpfwort (Du dumme Sau!), Adjektiv + Schimpfwort (Blöde Kuh!) oder Pronomen + Adjektiv (Du Ausgekochter!). Laut Scheffler (ebd.: 107) kann sich metaphorische Beschimpfung ,auf verschiedene Referenzobjekte beziehen: Gegenstände (Flegel), Pflanzen (Birne), Tiere (Kamel), Körperteile (Armleuchter) u. a.“. Sie hebt die Tierwelt als Quelle für zahlreiche auf Ähnlichkeitsbeziehung basierende Schimpfwörter hervor, denn „durch häufigen Gebrauch sind aus Tiermetaphern feste personale Bedeutungen, neue Sememe, entstanden, wodurch die Bedeutungsstrukturen dieser Lexeme erweitert wurden“" (ebd.: 108). Grygiel/Kleparski (2007: 89) sind der Meinung, dass Tiernamen an sich keine emotionale Aufladung aufweisen, aber wenn sie für die Benennung von Menschen verwendet werden, nehmen sie unterschiedliche negative Konnotationen an, und diese Konnotationen variieren in unterschiedlichen Sprachen. Zoonyme im Kroatischen sind expressiv und stellen eine Dauerquelle neuer Verwendung in der Rede dar, es entstehen immer neue Vergleiche, Metaphern und metonymische Bedeutungsveränderungen (Ljubičić 1994: 21).

Im Kroatischen und Deutschen sind einige Gemeinsamkeiten im Bereich des Ausdrucks von Pejoration vorhanden. Laut Stojić/Pavić Pintarić (2014: 354) werden in beiden Sprachen Tiernamen mit pejorativer Bedeutung für die Beschimpfung von Frauen (Ziege, Kuh, Gans, Eule, Schaf, Schlange) und Männern (Ochse, Esel, Affe, Schwein) verwendet. Neben diesen Bezeichnungen werden Adjektive als Intensivierer und Bezeichnungen der Eigenschaft, die beschimpft wird, den Pejorativa zugefügt, z. B. dumm.

Im Deutschen werden Zusammensetzungen mit pejorativer Bedeutung verwendet, z. B. mit den Konstituenten Scheiß-, Mist-, Sau-Dreck-, während im Kroatischen Zusammensetzungen selten vorkommen. Eine Gruppe bilden Zusammensetzungen, deren Grundwort ein Verb ist, während sich das Bestimmungswort auf das Referenzobjekt bezieht. Sie haben vor allem eine negative Bedeutung, z. B. ispičutura, tužibaba (vgl. Babić 2002: 373-374). Aus dem pejorativ konnotierten Phrasem cijepati dlaku ,zimperlich sein“ wurde cjepidlaka und aus lizati kome dupe, sich einschmeicheln` dupelizac abgeleitet. 
Die Ableitung der Pejorativa wird im Deutschen mit Morphemen wie -ler (Versöhnler, Abweichler) und -ling (Feigling, Mischling) oder Präfixen und Suffixen wie in Gesinge, Gejammere durchgeführt (Stojić/Pavić Pintarić 2014: 345). Vornehmlich werden im Kroatischen Pejorativa mit augmentativen Suffixen aus Substantiven abgeleitet, so -etina (babetina), -urina (zmijurina), -ina (konjina). Bei Bildungen auf -ina ist aber der Kontext zur Bestimmung der emotiven Bedeutung entscheidend, denn manchmal können diese Substantive als Hypokoristika verwendet werden (vgl. Babić 2002: 256). Einige Pejorativa werden aus Verben mit dem Suffix -alo (für Bezeichnungen im Neutrum, z. B. cendralo, njurgalo, baljezgalo) gebildet, oder auch mit -ac, nachdem aus den Verben ein Adjektiv abgeleitet wurde, z. B. brbljavac, cendravac. Diese Bildungen sind emotional in einem pejorativen Sinne markiert. Sie werden aus Verben gebildet, die eine gesellschaftlich schlechte oder negative Handlung bezeichnen (vgl. Babić 2002: 300). Das Suffix -ac kommt bei Ableitungen aus Adjektiven vor, z. B. ljigavac, traljavac. Eine Frau mit großem Hintern wird mit pejorativen Substantiven guzara und dupetara bezeichnet, die mit dem augmentativen Suffix -ara gebildet werden. Neben Suffixen zur Bildung von Augmentativen mit pejorativer Bedeutung dient im Kroatischen auch die Diminutivbildung zur Pejoration, z. B. čovječuljak, junačić.

Beide Sprachen verfügen über pejorative syntaktische Konstruktionen wie $D u / S i e X$ ! (Du Esel!), Adjektiv $+X$ (Alte Hexe!), Du bist/Sie sind + Adjektiv (Du bist blöd!) oder So ein X (So ein Aas) im Deutschen und $X$ jedan! (Konju jedan!), Pravi si X! (Pravi si krmak!), Jesi pravi X! (Jesi pravi magarac!) oder Baš si X! im Kroatischen (vgl. Stojić/Pavić Pintarić 2014: $345)$.

In beiden Sprachen werden auch metonymische Verschiebungen als Pejorativa verwendet, wie z. B. im Deutschen Schafskopf bzw. im Kroatischen glavonja (< Kopf), guzonja ( $<$ Hintern), mudonja (< Hoden) oder Adjektive aus Tierkörperteilen wie krakat ( $<$ Arme der Kraken). Dabei ist die Metonymie im Kroatischen stärker ausgedrückt, da die Personenbezeichnung von einem Körperteil gebildet wird.

In diesem Kapitel wurde gezeigt, dass beide Sprachen ihre Pejorativa häufig aus der Tierwelt nehmen, und dass bei der Pejoration die Metapher häufig eine wichtige Rolle spielt.

\section{3 Übersetzung der Pejorativa als Metapher}

Bedeutungsbereiche, denen die Pejorativa in den untersuchten Novellen entstammen sind: Charaktereigenschaften (fakin, gad, hulja, lopov, pacer, šeprtlja, tat), Intellekt (diletant, glupan, idiot, zalupanec), Berufe (balerina, kelner, lugar, mežnar, vatrogasac) und Zoonyme (gamad, konj, marva, mulac, pseto, skunks, som, stjenica, svinja, štakori, tele, tvor, vol, žohar). Absolute pejorative Personenbezeichnungen können unserem Korpus nach wörtlich übersetzt werden, z. B. pacer, idiot, diletant, neznalica (Patzer, Idiot, Dilettant, Ignorant). Bei der Übersetzung der Berufe und Zoonyme muss aber auf das metaphorische Potenzial der deutschen und kroatischen Sprache geachtet werden. Skirl/Schwarz-Friesel sind der Meinung, 
Metaphern eignen sich [...] in hohem Maße zur Vermittlung von Werturteilen, also zur Evaluation, wenn mit den Konzepten, auf die die metaphorisch verwendeten Ausdrücke referieren, konventionell eine bestimmte positive oder negative Bewertung assoziiert wird. [...] Werden die Bewertungen von den Rezipienten geteilt, so können die Metaphern bei ihnen ähnliche Emotionen auslösen, also die Funktion der Emotionalisierung erfüllen.

(2013: 63)

Al-Hasnawi (2007) schreibt über die Übersetzung von Metaphern in Bezug auf unterschiedliche Kulturen. Kultur beinhaltet u. a. Wissen, Glauben, Moral, Recht, Gewohnheiten und Wertesysteme, die ein Mensch als Mitglied einer Gemeinschaft erworben hat. ${ }^{6}$ Er nennt die Ansichten von Lakoff/Johnson (1980: 12), die behaupten, dass die Kultur u. a. verfügbare Metaphern anbietet, um die Realität verständlich zu machen. Mitglieder einer Kulturgemeinschaft verwenden die Sprache, um ihre Einstellungen zur Welt und zur Gemeinschaft auszudrücken. Eben darin liegt die Komplexität der Übersetzung von Metaphern. Für Dagut (1976) sind Metaphern zutiefst kulturspezifisch und wirken in einem Text schockierend für den Leser. Er argumentiert, dass keine allgemeinen Regeln zur Übersetzung von Metaphern bestehen, sondern dass sie von bestimmten kulturellen Erfahrungen und semantischen Assoziationen sowie von der Möglichkeit, die Metaphern in der Zielsprache zu reproduzieren, abhängen (ebd.: 32). Newmark (1988: 105) findet, dass bei der Übersetzung von Metaphern die Quelle des Vergleichs erkannt werden sollte. Dazu sollte auch verstanden werden, ob der Vergleich konnotativ oder denotativ ist. Er unterscheidet sieben Strategien für die Metaphernübersetzung (ebd.: 107): Reproduktion des gleichen Bildes (image) in der Zielsprache (ZS); Ersetzung des Bildes in der Ausgangssprache (AS) mit dem üblichen Bild in der ZS; Umwandlung der Metapher in einen Vergleich; Übersetzung der Metapher mit einem Vergleich und Sinn; Angabe des Sinns durch nichtmetaphorischen Ausdruck (Schäffner 2003: 282); Weglassen; Kombination der gleichen Metapher mit dem Sinn. „Die Wahl des Übersetzungsverfahrens hängt von verschiedenen Faktoren ab, vor allem von der Funktion der Metapher im Text, von stilistischen Erwägungen, von Konventionen der Textsorte sowie vom Übersetzungsauftrag“ (Schäffner 2003: 283). Darauf folgend hebt Schäffner kulturelle Unterschiede als das oft angeführte Problem für die Metaphernübersetzung hervor,

z. B. weil bestimmte Metaphern andere Assoziationen auslösen oder weil der Quellenbereich der AS-Metapher in der Soziokultur der ZS-Gemeinschaft keine (bedeutende) Rolle spielt. Das wird besonders deutlich bei Tiermetaphern [...]. Deshalb wird oft empfohlen, in solchen Fällen auf eine wörtliche Übersetzung zu verzichten und entweder eine angemessene ZS-Metapher zu verwenden oder den Sinn der AS-Metapher mittels einer Paraphrase wiederzugeben.

(ebd.: 284)

Havryliv (2009: 165-167) nennt kulturelle Tabus, Wertesysteme und stereotype Vorstellungen, die bei der Übersetzung der Schimpfwörter als problematisch identifiziert wurden, denn dabei sollte nicht die begriffliche Seite der Schimpfwörter, sondern die emotive Bedeutung eine wichtige Rolle spielen. Bei der Suche nach den entsprechenden Pejorativa sollte Folgendes beachtet werden: Die Intensität der pejorativen Lexeme sollte in beiden Sprachen übereinstimmen; Berücksichtigung der Etymologie; Häufigkeit des Gebrauchs; stilistische Färbung sollte in beiden Sprachen im Einklang sein. Dazu sollte bei der Übersetzung der metaphori-

\footnotetext{
${ }^{6}$ Snell-Hornby (1995: 41) bezieht u. a. die Einbettung der Kultur in einem Text auf die Übersetzungsmöglichkeit eines Textes.
}

ISSN 1615-3014 
schen Pejorativa das tertium comparationis in beiden Sprachen übereinstimmen, z. B. tertium comparationis - dick, Tiermetapher - Kuh; dick, schmutzig - Schwein, usw.

Zusammenfassend lässt sich feststellen, dass negative Konnotationen der Pejorativa in einem emotionalen Kontext zum Ausdruck kommen. Sprachen unterscheiden sich nach den Metaphern, die dabei hervorgerufen werden, was heißt, dass bei der Übersetzung das tertium comparationis der AS und ZS verglichen werden sollte.

\section{$4 \quad$ Analyse}

Die Übersetzungsanalyse umfasste 47 Ärgerausbrüche der Offiziere in den Dialogen. Dabei werden vor allem relative Pejorativa verwendet, und zwar Tiermetaphern. Mit den Pejorativa sind Emotionen verbunden, die häufig im einleitenden Teil des Gesagten, d. h. in der Beschreibung des nonverbalen Verhaltens, ausgedrückt sind. Für diesen Beitrag war von Interesse, mit welchen Emotionen Pejorativa verbunden werden, ob sie in einem bestimmten Grad ausgedrückt werden, und ob diese Aspekte des AT auch im ZT beibehalten wurden.

Die Beleidigung im ersten Beispiel ist in beiden Texten zu finden. Im Kroatischen ist das nonverbale Verhalten in der Vergangenheit ausgedrückt, wobei die emotionale Aufregung mit der Wiederholung des iterativen Verbs vozikam ,herumfahren, hin und her fahren' betont wird. Die Frau beschimpft ihren Gesprächspartner als lopov ,Dieb“ und zwar mit der Negation nijedan ,keiner', was die Beleidigung noch verstärkt. Dabei wird die expressive Wortfolge verwendet: zuerst das Pejorativum und dann die Verstärkung. In der deutschen Übersetzung wurde dafür Präsens ohne die Wiederholung des Verbs herumreisen verwendet. Dieb ist als Halunke übersetzt, also als ein schlechter, gemeiner Mensch. Durch die Transposition wurden grammatische Eigenschaften aus dem AT geändert. Das tertium comparationis ist aber gleich - ein schlechter Mensch.

1) AT: Žena se uzvrpoljila. Povrijedio je grubi ton: Što se vozikam? Što se vozikam? A što je tebe to briga, lopove nijedan? (Krleža 1985: 294)

ZT: Die Frau wird unruhig. Der grobe Ton beleidigt sie: Warum ich herumreise? Was geht das denn dich an, du Halunke? (Krleža 1984: 376)

Im zweiten Beispiel sind Emotionen, die zur Beschimpfung führen, wieder in der Einleitung der direkten Rede angegeben. Wegen der Hilflosigkeit seines Kollegen wird der Offizier wütend und beschimpft den Soldaten als kljasti kramar, romar und tele. Kljasti bezeichnet eine körperbehinderte Person; romar ist die Bezeichnung für einen Pilger nach Rom. Im Kroatischen befindet sich die Beschimpfung to tele , dieses Kalb“ am Ende des Satzes, als Hervorhebung des Ärgers. In der Übersetzung wurde die Bezeichnung romar als Maria-FeistritzPilger, also als kulturelle Substitution verwendet. Das Pejorativum Kalb erscheint am Anfang des Satzes und fungiert als Subjekt.

2) AT: Kada je ugledao satniju [...], pa Schwarza, gdje se bespomoćno vrze pred linijom, nešto je trglo njime i nije mogao da ostane neutralan. - Valjda ne ću tom kljastom kramaru, tom romaru dozvoliti da na moje oči kvari materijal, s kojim sam tako dugo i krvavo radio. Da mi još uništi cijeli posao posljednji dan, to tele! (ebd.: 42) 
ZT: Als er dann die Kompanie [...] erblickte, und Leutnant Schwarz vor der Linie hilflos herumschlich, ließ ihn etwas auffahren, und er konnte nicht ruhig bleiben. Ich werde doch nicht diesem verstümmelten Krämer, diesem MariaFeistritz-Pilger gestatten, daß er vor meinen Augen das Material verdirbt, mit dem ich so lange und blutig gearbeitet habe. Daß mir dieses Kalb noch am letzten Tag meine ganze Arbeit vernichtet! (ebd.: 52)

In folgenden Beispielen ist die Pejoration mit Adjektiven verstärkt. Im Beispiel (3) ist ein okkasionelles Adjektiv vom Substantiv gebildet. Das Kroatische verfügt über diese Möglichkeit, die hier zur Betonung der schlechten Eigenschaft dient. Marva bezeichnet Vieh für die Ackerarbeit. In der Übersetzung wird an dieser Stelle Hund verwendet. Das tertium comparationis ist beibehalten; marva bezieht sich auf Menschen, die nicht zu ehren sind, die kein Gefühl für das, was sie tun oder für andere Menschen haben. Hund bezeichnet auch gemeine Menschen.

3) AT: Ruka ostaje gore, marvo marvasta! (ebd.: 130)

ZT: Die Hände bleiben oben, blöder Hund! (ebd.: 165)

Im Beispiel (4) wurde das neugebildete Adjektiv svinjast als schweinisch übersetzt. Dazu wird im ZT das Substantiv Schweine zweimal wiederholt, da die erste Beleidigung mrcine vi lopovske wörtl. ,Luder ihr diebischen' mit dem Personalpronomen vi ,ihr' zwischen zwei Pejorativa, nur als Gauner übersetzt wurde. Die Intensität ist aber mit der Wiederholung des Substantivs Schweine beibehalten.

4) AT: [...] Mrcine vi lopovske! Svinje svinjske! (ebd.: 110)

ZT: [...] Gauner! Schweine! Schweinische Schweine! (ebd.: 145)

Im folgenden Beispiel wird die Bezeichnung lazari in der Übersetzung erklärt. Das Adjektiv prokleti, verdammte' signalisiert, dass das Substantiv als ein Pejorativum verwendet wird.

5) AT: Na, ovi prokleti lazari ovdje! Jesu li to domobrani? (ebd.: 53)

ZT: Diese verdammten Kerle hier - armselig wie Lazarus! Sind das denn Honveds? (ebd.: 68)

In den Kombinationen der Pejorativa und Adjektive kann man im Kroatischen bemerken, dass die Struktur der pejorativen Ausdrücke häufig die folgende ist: Substantiv (Personenbezeichnung) + Adjektiv (Intensivierer). Im Deutschen ist diese expressive Wortreihe nicht möglich, und sie wird als Adjektiv (Intensivierer) + Substantiv (Personenbezeichnung) realisiert. Dies ist besonders im Beispiel (6) zu bemerken: svinja ušljiva, ti some gnjili, crvljivi. Das zweite Adjektiv crvljivi ,madig' wurde im ZT weggelassen. Im AT signalisiert die Nachreihung von zwei Adjektiven, die mit dem Wort Wels normalerweise nicht im gleichen Kontext verwendet werden, den hohen Grad der Aufregung.

6) AT: Mir! Ne gibaj se! Svinja ušljiva! Što se mrijestiš, ti some gnjili, crvljivi! Ti! (ebd.: 45)

ZT: Still! Rühr dich nicht! Lausiges Schwein! Was lachst du, du verfaulter Wels, du? (ebd.: 57) 
In der expressiven Wortreihe wird mit der Beleidigung häufig das Personalpronomen $t i$,du oder $v i$,ihr' verwendet, wenn mehrere Soldaten gemeint sind. Dies führt zur Intensivierung der Beleidigung.

7) AT: Marš, ti tat zagorski! Svi ste vi tati! - Nisam ja tat, nego ste vi meni moje pravo ukrali! (ebd.: 16)

ZT: Marsch, du Zagorianer Dieb! Ihr seid alle Diebe! - Ich bin kein Dieb, sondern Sie haben mir mein Recht gestohlen! (ebd.: 19)

AT: No! Što je to? Zar ja uzalud govorim? Jesam li ja vaš gratulant, pa vam deklamiram, vi svinje zagorske? (ebd.: 109)

ZT: Rede ich denn umsonst? Habt ihr nichts zu tun, ihr verdammten Zagorianer Schweine? (ebd.: 144)

Die expressive Wortreihe mit dem nachgestellten Attribut kann noch mit dem Dativus ethicus hervorgehoben werden: mater ti ludu wörtl. ,Mutter dir verrückte', wobei die Übersetzung die Verfluchung ausdrücklich nennt.

8) AT: Mater ti ludu! Ustrijelit će te kao psa! Marš! (ebd.: 25)

ZT: Verflucht sei deine verrückte Mutter! Sie werden dich wie einen Hund niederschießen! Marsch! (ebd.: 32)

Im Kroatischen wird majka/mater in Schimpfwörtern als Verstärkung verwendet, was in der Übersetzung im Beispiel (9) als verdammt übersetzt wurde. Soldaten werden im AT als schlapp beschrieben, sie werden wie Weiber ausgelacht. Im ZT werden Weiber noch als alt bezeichnet. Im AT kann kokošar einen Hühnerdieb aber auch einen Mann bezeichnen, der Hühner züchtet.

9) AT: Kada vas ugledaju Čerkezi i Kozaci, smijat će vam se kao babama. [...] Kozaci nisu šeprtlje, majku vam kokošarsku! (ebd.: 75)

ZT: Wenn euch die Tscherkessen und Kosaken zu Gesicht bekommen, werden sie über euch lachen wie über alte Weiber! [...] Die Kosaken sind keine Pfuscher! Zum Teufel mit euch, ihr verdammten Hühnerdiebe! (ebd.: 99)

Das Wort mrcina bezeichnet im Kroatischen einen starken, korpulenten Mann, aber auch pejorativ einen wertlosen, faulen Mann. Im ZT wurde das Substantiv als Vieh übersetzt, was rohe, brutale Menschen bezeichnet.

10) AT: Čuješ ti, mrcina! [...] Udri da sve puca! - [...] Čuješ ti! Lopov prokleti! Opet simuljeraš! (ebd.: 224)

ZT: Hörst du, Vieh! Hau zu, daß alles kracht! - [...] Hörst du! Verdammter Gauner! Wieder simulierst du! (ebd.: 287)

Im Zusammenhang mit dem Pejorativum mulac ,Maulesel' werden Soldaten auch als Ballerinnen bezeichnet. 
11) AT: No! Kaj bu? Em niste balerine! Mulci prokleti! Trkom! (ebd.: 24)

ZT: Was ist los? Seid ihr denn Ballerinen? Verfluchte Maulesel, Laufschritt! (ebd.: 30)

Im Beispiel (12) wird die Bezeichnung tat Kristušov als Kirchendieb übersetzt. Hier kann man diese Bezeichnung als einen Dieb, der zum Christ gehört, verstehen. Der Balken (bolvan) wird auch als gehörend zum Gott bezeichnet. In der Übersetzung ist die Zusammensetzung Kirchendieb und statt Balken die Strafe Gottes verwendet. Balvan ,Balken' steht oft für dumme Personen, was in der Übersetzung mit der weniger expressiven Strafe Gottes übertragen wurde.

12) AT: Čuješ, ti! Gad leni! Amo čašu vode! -Čuješ, ti! Tat Kristušov! [...] Si razmel, bolvane božji? (ebd.: 224)

ZT: Hörst du! Faules Biest! Her mit einem Glas Wasser! -Hörst du! Kirchendieb! [...] Hast du verstanden, du Strafe Gottes? (ebd.: 287)

Das folgende Beispiel zeigt mit der Silbentrennung im AT, dass Offiziere schreien, was auch in der Beschreibung des nonverbalen Verhaltens zu lesen ist. Im ZT ist dieses Schreien nicht orthographisch dargestellt. Mulec (dialektal für mulac, ,Maulesel') ist im ZT durch verdammt verstärkt. Dafür wird svetec (svetac, ,Heiliger') im ZT weggelassen, und svinja gubava, lepröses Schwein` ist als verdammtes Schwein übersetzt. Man könnte daraus schließen, dass die Übersetzung weniger expressiv ist, obwohl das tertium comparationis (Maulesel und Schwein) in beiden Sprachen übereinstimmt.

13) AT: Jedan.dva, jedan-dva - viču gospoda potčasnici iz svega grla i udaraju ljude kundacima pod rebra, guraju ih šakom u leđa, [...] - Jedan-dva! Jam-brek! [...] Mu-lec! Sve-tec! Gla-vu van! [...] Svi-nja gu-ba-va! (ebd.: 61)

ZT: $\quad$ Eins - zwei! Eins - zwei! - schreien die Herren Unteroffiziere aus voller Kehle und schlagen die Leute mit den Kolben in die Rippen, stoßen sie mit den Fäusten in den Rücken [...]. - Eins - zwei! Eins - zwei! Jam-brek! [...] Verdammter Maulesel! Kopf hoch! Bauch hinein! Verdammtes Schwein! Eins - zwei! Eins - zwei! (ebd.: 79)

\section{$5 \quad$ Schlussfolgerung}

In diesem Beitrag wurde die Übersetzung der Pejorativa aus dem Kroatischen ins Deutsche untersucht. Pejorativa wurden als emotionale Elemente der Rede betrachtet. Vorgesetzte benutzten sie in emotional aufgeladenen Situationen, meistens beim Ärgerausbruch, als Beleidigungsmittel. Absolute Pejorativa beziehen sich auf Charaktereigenschaften (fakin, gad, hulja, lopov, pacer, šeprtlja, tat) und den Intellekt (diletant, glupan, idiot, zalupanec), während relative Pejorativa den Bereichen der Berufe (Balletttänzerin, Feuerwehr, Kellner, Krämer, Waldhüter) und der Zoonyme (Aas, Iltis, Kalb, Ochse, Pferd, Ratten, Schabe, Schwein, Skunks, Ungeziefer, Vieh, Wals, Wanze) entstammen. Sie werden mit Personal- und Demonstrativpronomen ( $d u$, ihr, diese) sowie negativ konnotierten attributiven Adjektiven (v. a. faul, lausig, verdammt) verbunden. 
Die Verwendung der Pejorativa hängt mit den im Text ausgedrückten Emotionen zusammen, die in literarischen Texten vor allem in der Einleitung der Rede, d. h. in der Beschreibung des nonverbalen Verhaltens angedeutet werden. Absolute Pejorativa können aus dem Kroatischen ins Deutsche eindeutig übersetzt werden, da sie nur diese pejorative Bedeutung tragen, z. B. lopov, tat $\rightarrow$ Dieb, Gauner, Halunke, glupan $\rightarrow$ Dummkopf, diletant $\rightarrow$ Dilettant, pacer $\rightarrow$ Patzer, neznalica $\rightarrow$ Ignorant, Idiot $\rightarrow$ Idiot, bagra $\rightarrow$ Bagage, šmrkavac $\rightarrow$ Rotzbub, fakin $\rightarrow$ Spitzbub, Gauner, zalupanec $\rightarrow$ Trottel.

Relative Pejorativa sind eine Herausforderung für die Übersetzer, die ihre pejorative Bedeutung im AT sowie das tertium comparationis in beiden Sprachen kennen sollten. Dabei spielt unserer Ansicht nach die Einleitung der Pejoration eine Rolle, z. B. Čuješ ti! ,Hörst du! ‘, Zar ja uzalud govorim? ,Rede ich denn umsonst?' Mař̌! ,Marsch!‘, Kuda trčiš? ,Wohin rennst du?', Ǐs, iš (...)! ,Scht, scht‘, Mir! ,Still!‘, Kakva je to glupost? ,Was sind das für Dummheiten?‘. In diesen kurzen Äußerungen erkennt man die Ungeduld und Aufregung, die im weiteren Text zu Pejoration führen. Unter den relativen Pejorativa wurden nach Translationsmöglichkeiten drei Gruppen festgestellt. In der ersten Gruppe befinden sich Tiernamen und Berufe, die eine Entsprechung im Deutschen haben, z. B. vol $\rightarrow$ Ochse, svinja $\rightarrow$ Schwein, bik $\rightarrow$ Stier, konji $\rightarrow$ Pferde, krava $\rightarrow$ Kuh, marva $\rightarrow$ Rindviecher, pilići $\rightarrow$ Kücken, som $\rightarrow$ Wels, štakori $\rightarrow$ Ratten, tele $\rightarrow$ Kalb, tvor $\rightarrow$ Stinktier, kelner $\rightarrow$ Kellner, mežnar $\rightarrow$ Mesner, vatrogasci $\rightarrow$ Feuerwehrleute.

Die zweite Gruppe bilden Wörter, bei deren Übersetzung die Metapher übertragen werden sollte, die in der deutschen Sprache eine entsprechende Bedeutung hat; man sucht also nach funktionalen Äquivalenten. So wurde das Substantiv mrcina ,Luder' als Gauner und Vieh, mulac als Esel oder Maulesel übersetzt. Zu dieser Gruppe gehört auch die Bezeichnung nesrećo ,Unglück', die im Kroatischen in beleidigenden Kontexten verwendet wird. Im ZT ist die Bezeichnung Unglückswurm belegt.

Die dritte Gruppe umfasst Pejorativa, die entweder erklärt oder mit einer kulturellen Substitution übersetzt werden. Pejorativ konnotierte Substantive wurden im ZT erklärt, wie z. B. la$z a r i \rightarrow$ Kerle... armselig wie Lazarus. Andere werden mit der kulturellen Substitution übersetzt, z. B. romar $\rightarrow$ Maria-Feistritz-Pilger: romar bezieht sich auf den Pilger nach Rom und Maria-Feistritz ist ein Wallfahrtsort in Österreich.

Relative Pejorativa werden mit Adjektiven verwendet, die signalisieren, dass es sich um eine Beleidigung handelt und die diese zugleich verstärken. Bei der Übersetzung werden Adjektive in den meisten Fällen ins Deutsche übertragen. So wurden bei der Analyse folgende Kombinationen belegt, z. B. mulci prokleti $\rightarrow$ verfluchte Maulesel, Svinja ušljiva! $\rightarrow$ Lausiges Schwein! Im Kroatischen werden sie in der expressiven Wortreihe ausgedrückt, was in der deutschen Übersetzung nicht übertragen werden kann.

Im ZT findet man auch andere Adjektive, die die pejorative Bedeutung des Substantivs betonen, z. B. svinja gubava, lepröses Schwein', wobei die Übersetzung verdammtes Schwein lautet, da Lepra auf Menschen bezogen wird.

Es gibt auch Beispiele, wo das Pejorativum in der Übersetzung durch das zugefügte Adjektiv noch verstärkt wurde, z. B. vi svinje zagorske, ihr Zagorianer Schweine“ $\rightarrow$ ihr verdammten Zagorianer Schweine. 
Im Kroatischen kann man Adjektive aus den Substantiven bilden, um ihre Bedeutung zu betonen, z. B. marvo marvasta oder svinje svinjaste. Diese Neubildungen werden entsprechend übersetzt, wenn es in der ZS solche Adjektive gibt wie bei schweinische Schweine. Wenn es die aber nicht gibt, sucht man nach einem funktionalen Äquivalent.

Die Analyse hat gezeigt, dass Kroatisch und Deutsch über ähnliche Metaphern im Bereich der Pejorativa verfügen. Übersetzungsstrategien, die sich in dem untersuchten Korpus aufweisen, sind: funktionale Äquivalente, Explizierung, kulturelle Substitution, Transposition und Übersetzung mit einem weniger expressiven Wort. Es ist zu bemerken, dass kroatische Adjektive, die die Pejorativa näher bezeichnen oder verstärken, häufig mit dem Adjektiv verdammt, das im Deutschen Wut und Ärger ausdrückt und verstärkt, übersetzt werden. Dazu ist die Wortfolge in kroatischen Beleidigungen veränderbar.

Bei der Übersetzung der Pejorativa aus dem Kroatischen ins Deutsche oder umgekehrt soll darauf geachtet werden, welchen emotionalen Ton die Pejorativa dem Kontext verleihen, um die richtige Metapher oder das tertium comparationis in der ZS zu finden.

\section{Korpus}

Krleža, Miroslav (1985): Hrvatski bog Mars. Split: Logos.

Krleža, Miroslav (1984): Der kroatische Gott Mars. Erzählungen. Königstein: Athenäum (übers. von Milica Sacher-Masoch, Reinhard Federmann und Milo Dor).

\section{Literatur}

Al-Hasnawi, Ali R. (2007): “A Cognitive Approach to Translating Metaphors". Translation Journal 11/3. http://translationjournal.net/journal/41metaphor.htm. [20.09.2016].

Babić, Stjepan (2002): Tvorba riječi u hrvatskome književnome jeziku. Zagreb: Nakladni zavod Globus, Hrvatska akademija znanosti i umjetnosti.

Beller, Charley (2013): "Manufactured and inherent pejorativity". In: Snider, Todd (ed.): Proceedings of SALT 23. Santa Cruz, University of California: 136-155.

Bergerová, Hana (2015): „Ärger - zlost““. In: Bergerová, Hana et al. (eds.): Lexikalische Ausdrucksmittel der Emotionalität im Deutschen und im Tschechischen. Ostrava, Ostravská univerzita v Ostravě: 111-153.

Croom, Adam M. (2013): "How to do things with slurs. Studies in the way of derogatory words". Language \& Communication 33: 177-204.

Dagut, Menachem (1976): “Can 'metaphor' be translated”. Babel: International Journal of Translation 22/1: 21-33.

Duden (2003): Deutsches Universalwörterbuch. 5. Auflage. Mannheim: Dudenverlag.

Espunya, Anna/Pavić Pintarić, Anita (2016): „Identität und Emotionalität in Vázquez Montalbáns Carvalho Roman Los mares del sur und seinen deutschen Übersetzungen“. In: Pavić Pintarić, Anita et al. (eds.): Sprachliche Konstituierung der Identität durch Emotionalität. Tübingen, Narr Francke Attempto: 127-143.

Goetsch, Paul (1985): „Fingierte Mündlichkeit in der Erzählkunst entwickelter Schriftkulturen“. Poetica 17: 202-218.

Grygiel, Marcin/Grzegorz A. Kleparski (2007): Main Trends in Historical Semantics. Rzeszów: Wydawnictwo Uniwersytetu Rzeszowskiego. 
Havryliv, Oksana (2003): Pejorative Lexik. Untersuchungen zu ihrem semantischen und kommunikativ-pragmatischen Aspekt am Beispiel moderner deutschsprachiger, besonders österreichischer Literatur. Frankfurt a. M.: Lang.

Havryliv, Oksana (2009): Verbale Aggression. Formen und Funktionen am Beispiel des Wienerischen. Frankfurt a. M.: Lang.

Lakoff, Goerge/Johnson, Mark (1980): Metaphors We Live by. Chicago: University of Chicago Press.

Liimatainen, Annikki (2011): „Ach du Donnerchen! - Voi herran pieksut! Zur Wiedergabe der emotiven Formeln in deutschen und finnischen Übersetzungen“. In: Hyvärinenn, Irma/Liimatainen, Annikki (eds.): Beiträge zur pragmatischen Phraseologie. Frankfurt a. M. etc., Lang: 113-145. (= Finnische Beiträge zur Germanistik 25).

Ljubičić, Maslina (1994): „O hrvatskim zoonimima: konotativno značenje i frazeologija”. Filologija 22-23: 245-252.

Martin James Robert/White, Peter R. R. (2005): The Language of Evaluation. Appraisal in English. New York: Houndmills.

Newmark, Peter (1988): A textbook of translation. London/New York: Prentice Hall International.

O'Brien, Gerald V. (2009): "Metaphors and the pejorative framing of marginalized groups. Implications for social work education". Journal of Social Work Education 45/1: 29-46.

Pavić Pintarić, Anita (in Druck). „Emotionalität in Novellen von Miroslav Krleža“. In: Tvrdik, Milan/Haslmayr, Harald (eds.): Frieden und Krieg im mitteleuropäischen Raum. Historisches Gedächtnis und literarische Reflexion. Wien, new academic. (= Transkulturelle Forschungen an den Österreich-Bibliotheken im Ausland).

Rocco, Goranka/Pavić Pintarić, Anita (2016): „Übersetzung gesprochensprachlicher und dialektaler Elemente in der Erzählliteratur. Auf dem Weg zu einem translationsdidaktischen Modell“،. In: Udier, Sanda Lucija et al. (eds.): Zbornik radova s međunarodnoga znanstvenog skupa Hrvatskoga društva za primijenjenu lingvistiku Metodologija i primjena lingvističkih istraživanja. Zagreb, Srednja Europa: 251-264.

Schäffner, Christina (2003): „Metaphern“. In: Snell-Hornby, Mary et al. (eds.): Handbuch Translation. Tübingen, Stauffenburg: 280-285.

Scheffler, Gabriele (2000): Schimpfwörter im Themenvorrat einer Gesellschaft. Marburg: Tectum.

Skirl, Helge/Schwarz-Friesel, Monika (2013): Metapher. Heidelberg: Universitätsverlag Winter.

Snell-Hornby, Mary (1995): Translation Studies. An Integrated Approach. Amsterdam/Philadelphia: Benjamins.

Stojić, Aneta/Pavić Pintarić, Anita (2014): "Pejorative Nouns in Speech Act of Insulting as Expression of Verbal Aggression”. Croatian Journal of Philosophy 14/3: 343-356. 